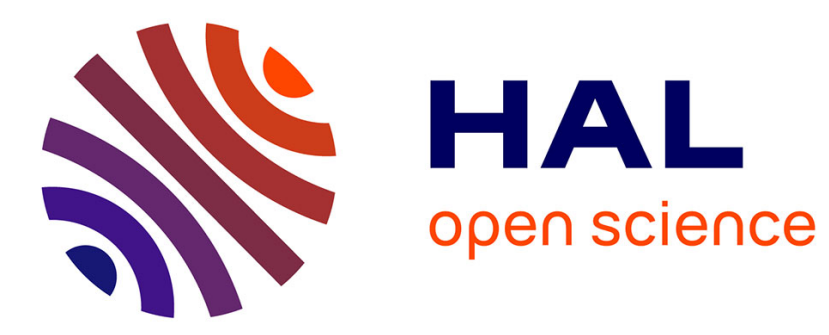

\title{
Robustness of delayed multistable systems
}

\author{
Denis Efimov, Johannes Schiffer, Romeo Ortega
}

\section{To cite this version:}

Denis Efimov, Johannes Schiffer, Romeo Ortega. Robustness of delayed multistable systems. G. Valmorbida; R. Sipahi; I. Boussaada; A. Seuret. Delays and Interconnections: Methodology, Algorithms and Applications, Springer, 2017, Lecture notes in Control and Information Sciences. hal-01632424

\section{HAL Id: hal-01632424 \\ https://hal-centralesupelec.archives-ouvertes.fr/hal-01632424}

Submitted on 23 Nov 2017

HAL is a multi-disciplinary open access archive for the deposit and dissemination of scientific research documents, whether they are published or not. The documents may come from teaching and research institutions in France or abroad, or from public or private research centers.
L'archive ouverte pluridisciplinaire HAL, est destinée au dépôt et à la diffusion de documents scientifiques de niveau recherche, publiés ou non, émanant des établissements d'enseignement et de recherche français ou étrangers, des laboratoires publics ou privés. 


\title{
Robustness of delayed multistable systems
}

\author{
Denis Efimov, Johannes Schiffer and Romeo Ortega
}

\begin{abstract}
In this chapter sufficient conditions for input-to-state stability (ISS) of delayed systems are presented using Lyapunov-Razumikhin functions. It is shown that ISS multistable systems are robust with respect to delays in the feedback path. First, the approach is illustrated by establishing the ISS property for the model of a nonlinear pendulum, then delay-dependent robustness conditions are derived. Second, it is shown that, under certain assumptions, the problem of phase-locking analysis in droop-controlled inverter-based microgrids with delays can be reduced to the stability investigation of a nonlinear pendulum, and corresponding delay-dependent conditions for asymptotic phase-locking are derived for an exemplary microgrid consisting of two droop-controlled inverters.
\end{abstract}

\section{Introduction}

The increasing penetration of renewable distributed generation (DG) units at the low and medium voltage levels has a strong impact on the power system structure [14, $45,13]$. This fact requires new control and operation strategies to ensure a reliable and efficient electrical power supply [14,17]. An emerging concept to address these

Denis Efimov

Inria, Non-A team, 40 avenue Halley, 59650 Villeneuve d'Ascq, France; CRIStAL (UMR-CNRS 9189), Ecole Centrale de Lille, Avenue Paul Langevin, 59651 Villeneuve d'Ascq, France; Department of Control Systems and Informatics, University ITMO, 49 avenue Kronverkskiy, 197101

Saint Petersburg, Russia, e-mail: denis.efimov@inria.fr

Johannes Schiffer

School of Electronic and Electrical Engineering, University of Leeds, Leeds LS2 9JT, UK, e-mail: j.schiffer@leeds.ac.uk

Romeo Ortega

Laboratoire des Signaux et Systémes, École Supérieure d'Electricité (SUPELEC), Gif-sur-Yvette 91192, France, e-mail: ortega@1ss.supelec.fr 
challenges is the microgrid [23, 20,14]. A microgrid is a locally controllable subset of a larger electrical network. It is composed of several DG units, storage devices and loads.

Typically, most DG units in an AC microgrid are connected to the network via AC inverters [17]. Under ideal conditions, an inverter-based DG unit can be modeled as an ideal controllable voltage source [24, 33]. Furthermore, a popular control scheme to operate inverter-based DG units with the purpose to achieve frequency synchronization and power sharing in the network is droop control [6, 19]. Conditions for stability in droop-controlled microgrids with inverters modeled as ideal controllable voltage sources have been derived, e.g., in [37, 35, 27].

However, in a practical setup, the droop control scheme is applied to an inverter by means of digital discrete time control. Besides clock drifts, see, e.g. [36], digital control usually introduces time delays [22, 25, 29]. According to [29], the main reasons for this are 1) sampling of control variables, 2) calculation time of the digital controller and 3) generation of the pulse-width modulation. We refer the reader to, e.g. [29] for further details. Hence, it is important to consider time delays in the stability analysis of microgrids.

In general, inverter-based microgrids operated with droop control have several equilibria [37, 35]. Thus they are multistable systems. Stability analysis $[4,12,44$, $26,28,31,32,34,38]$ and robust stability analysis $[1,3,5,7,42]$ for this class of systems is rather complicated. Recently, the ISS theory [40] has been extended to multistable systems in [2,3] (see also [21] for discussion on ISS property with respect to an unbounded set).

Motivated by the abovementioned phenomenon, the papers [11, 10] have extended the ISS framework for multistable systems $[2,3]$ to multistable systems with delay. In particular, sufficient conditions for ISS of multistable systems in the presence of delays are given in terms of a Lyapunov-Razumikhin function. It is also shown that ISS multistable systems are robust with respect to feedback delays (a simple but important illustration is via the example of a nonlinear pendulum). We would like to point out that related works on ISS of time-delay systems by employing Lyapunov functions $[8,16,30]$ are limited to systems with a single equilibrium point or a compact attracting set. Based on the results in $[11,10]$ (their detailed presentation is given below), in this chapter, a condition for asymptotic phase-locking in a microgrid composed of two droop-controlled inverters with delay is developed. The analysis is conducted for a simplified inverter model derived under the assumptions of constant voltage amplitudes and ideal clocks, as well as negligible dynamics of the internal inverter LC filter and controllers. In that scenario, the delay merely affects the phase angle of the inverter output voltage. The stability results are illustrated by simulations. 


\section{Preliminaries}

For an $n$-dimensional $\mathscr{C}^{2}$ connected and orientable Riemannian manifold $M$ without a boundary, let the map $f(x, d): M \times \mathbb{R}^{m} \rightarrow T_{x} M$ be of class $\mathscr{C}^{1}$, and consider a nonlinear system of the following form:

$$
\dot{x}(t)=f(x(t), d(t))
$$

where the state $x \in M$ and $d(t) \in \mathbb{R}^{m}$ (the input $d(\cdot)$ is a locally essentially bounded and measurable signal) for $t \geq 0$. We denote by $X\left(t, x_{0} ; d\right)$ the uniquely defined solution of (1) at time $t$ fulfilling $X\left(0, x_{0} ; d\right)=x_{0}$. Together with (1) we will analyze its unperturbed version:

$$
\dot{x}(t)=f(x(t), 0) .
$$

A set $S \subset M$ is invariant for the unperturbed system (2) if $X(t, x ; 0) \in S$ for all $t \in$ $\mathbb{R}$ and for all $x \in S$. Define the distance from a point $x \in M$ to the set $S \subset M$ as $|x|_{S}=\min _{a \in S} \delta(x, a)$, where the symbol $\delta\left(x_{1}, x_{2}\right)$ denotes the Riemannian distance between $x_{1}$ and $x_{2}$ in $M,|x|=|x|_{\{0\}}$ for $x \in M$ (in this case 0 represents a designated element of $M$ ) or a usual Euclidean norm of a vector $x \in \mathbb{R}^{n}$. For a signal $d: \mathbb{R} \rightarrow \mathbb{R}^{m}$ the essential supremum norm is defined as $\|d\|_{\infty}=\operatorname{ess~sup}_{t \geq 0}|d(t)|$.

\subsection{Decomposable sets}

Let $\Lambda \subset M$ be a compact invariant set for (2).

Definition 1. [28] A decomposition of $\Lambda$ is a finite and disjoint family of compact invariant sets $\Lambda_{1}, \ldots, \Lambda_{k}$ such that

$$
\Lambda=\bigcup_{i=1}^{k} \Lambda_{i} .
$$

For an invariant set $\Lambda$, its attracting and repulsing subsets are defined as follows:

$$
\begin{aligned}
& \mathfrak{A}(\Lambda)=\left\{x \in M:|X(t, x, 0)|_{\Lambda} \rightarrow 0 \text { as } t \rightarrow+\infty\right\}, \\
& \mathfrak{R}(\Lambda)=\left\{x \in M:|X(t, x, 0)|_{\Lambda} \rightarrow 0 \text { as } t \rightarrow-\infty\right\} .
\end{aligned}
$$

Define a relation on $\mathscr{W} \subset M$ and $\mathscr{D} \subset M$ by $\mathscr{W} \prec \mathscr{D}$ if $\mathfrak{A}(\mathscr{W}) \cap \mathfrak{R}(\mathscr{D}) \neq \oslash$.

Definition 2. [28] Let $\Lambda_{1}, \ldots, \Lambda_{k}$ be a decomposition of $\Lambda$, then

1. An $r$-cycle $(r \geq 2)$ is an ordered $r$-tuple of distinct indices $i_{1}, \ldots, i_{r}$ such that $\Lambda_{i_{1}} \prec \ldots \prec \Lambda_{i_{r}} \prec \Lambda_{i_{1}}$.

2. A 1-cycle is an index $i$ such that $\left[\Re\left(\Lambda_{i}\right) \cap \mathfrak{A}\left(\Lambda_{i}\right)\right]-\Lambda_{i} \neq \oslash$.

3. A filtration ordering is a numbering of the $\Lambda_{i}$ so that $\Lambda_{i} \prec \Lambda_{j} \Rightarrow i \leq j$. 
As we can conclude from Definition 2, existence of an $r$-cycle with $r \geq 2$ is equivalent to existence of a heteroclinic cycle for (2) [18]. Furthermore, existence of a 1-cycle implies existence of a homoclinic cycle for (2) [18].

Definition 3. The set $\mathscr{W}$ is called decomposable if it admits a finite decomposition without cycles, $\mathscr{W}=\bigcup_{i=1}^{k} \mathscr{W}_{i}$, for some non-empty disjoint compact sets $\mathscr{W}_{i}$, which form a filtration ordering of $\mathscr{W}$, as detailed in definitions 1 and 2 .

\subsection{Robustness notions}

The following robustness notions for systems represented by (1) have been introduced in [2,3] (see also [9] for a survey on the ISS framework).

Definition 4. We say that the system (1) has the practical asymptotic gain (pAG) property if there exist $\eta \in \mathscr{K}_{\infty}$ and a non-negative real $q$ such that for all $x \in M$ and all measurable essentially bounded inputs $d(\cdot)$ the solutions are defined for all $t \geq 0$ and the following holds:

$$
\limsup _{t \rightarrow+\infty}|X(t, x ; d)|_{\mathscr{W}} \leq \eta\left(\|d\|_{\infty}\right)+q .
$$

If $q=0$, then we say that the asymptotic gain (AG) property holds.

Definition 5. We say that the system (1) has the limit property (LIM) with respect to $\mathscr{W}$ if there exists $\mu \in \mathscr{K}_{\infty}$ such that for all $x \in M$ and all measurable essentially bounded inputs $d(\cdot)$ the solutions are defined for all $t \geq 0$ and the following holds:

$$
\inf _{t \geq 0}|X(t, x ; d)|_{\mathscr{W}} \leq \mu\left(\|d\|_{\infty}\right) .
$$

Definition 6. We say that the system (1) has the practical global stability (pGS) property with respect to $\mathscr{W}$ if there exist $\beta \in \mathscr{K}_{\infty}$ and $q \geq 0$ such that for all $x \in M$ and measurable essentially bounded inputs $d(\cdot)$ the following holds for all $t \geq 0$ :

$$
|X(t, x ; d)|_{\mathscr{W}} \leq q+\beta\left(\max \left\{|x|_{\mathscr{W}},\|d\|_{\infty}\right\}\right) .
$$

It has been shown in $[2,3]$ that to characterize pAG property in terms of Lyapunov functions the following notion is appropriate.

Definition 7. We say that a $\mathscr{C}^{1}$ function $V: M \rightarrow \mathbb{R}$ is a practical ISS-Lyapunov function for (1) if there exists $\mathscr{K}_{\infty}$ functions $\alpha_{1},\left[\alpha_{2}\right], \alpha_{3}$ and $\gamma$, and scalar $q \geq 0$ [and $c \geq 0$ ] such that

$$
\alpha_{1}\left(|x|_{\mathscr{W}}\right) \leq V(x) \leq\left[\alpha_{2}\left(|x|_{\mathscr{W}}+c\right)\right],
$$

the function $V$ is constant on each $\mathscr{W}_{i}$ and the following dissipation holds:

$$
D V(x) f(x, d) \leq-\alpha_{3}\left(|x|_{\mathscr{W}}\right)+\gamma(|d|)+q .
$$

If the latter inequality holds for $q=0$, then $V$ is said to be an ISS-Lyapunov function. 
Notice that $\alpha_{2}$ and $c$ are in square brackets as their existence follows (without any additional assumptions) by standard continuity arguments.

The main result of $[2,3]$ connecting these robust stability properties is stated below. It extends the results of $[39,41]$ obtained for connected sets.

Theorem 1. Consider a nonlinear system as in (1) and let a compact invariant set containing all $\alpha$-and $\omega$-limit sets of (2) $\mathscr{W}$ be decomposable (in the sense of Definition 3). Then the following facts are equivalent.

1. The system admits an ISS Lyapunov function;

2. The system enjoys the AG property;

3. The system admits a practical ISS Lyapunov function;

4. The system enjoys the $p A G$ property;

5. The system enjoys the LIM property and the $p G S$.

Definition 8. [3] Suppose that a nonlinear system as in (1) satisfies the assumptions and the list of equivalent properties of Theorem 1 . Then this system is called ISS with respect to the set $\mathscr{W}$.

\section{Multistable systems with delays}

Let $\tau>0$, for a function $d:[-\tau,+\infty) \rightarrow \mathbb{R}^{m}$ and $t \geq 0$ denote a function $d_{t}(\cdot)$ : $[-\tau, 0] \rightarrow \mathbb{R}^{m}$ defined by $d_{t}(\theta)=d(t+\theta)$ for $\theta \in[-\tau, 0]$. Denote by $\mathscr{D}$ a set of bounded and piecewise continuous functions $d_{t}(\cdot):[-\tau, 0] \rightarrow \mathbb{R}^{m}$. Consider a functional differential equation on an $n$-dimensional $\mathscr{C}^{2}$ connected and orientable Riemannian manifold $M$ without a boundary:

$$
\dot{x}(t)=F\left(x_{t}, d_{t}\right), x_{0} \in \mathscr{C}_{\tau},
$$

where the map $F: \mathscr{C}_{\tau} \times \mathscr{D} \rightarrow T_{x} M$ is of class $\mathscr{C}^{1}$ (we will denote a set of continuous functions $\xi:[-\tau, 0] \rightarrow M$ by $\left.\mathscr{C}_{\tau}\right), x(t) \in M$ is the state, $x_{t} \in \mathscr{C}_{\tau}$ and $d_{t} \in \mathscr{D}$ for all $t \geq 0$. We denote by $X\left(t, x_{0} ; d\right)$ the uniquely defined solution of (3) at time $t$ fulfilling $X\left(\theta, x_{0} ; d\right)=x_{0}(\theta)$ for all $\theta \in[-\tau, 0] ; X_{t}^{x_{0}, d}(\theta)=X\left(t+\theta, x_{0} ; d\right)$ for $\theta \in[-\tau, 0]$. Define as in [43]

$$
\left|x_{t}\right|=\max _{\theta \in[-\tau, 0]}|x(t+\theta)|,|| x||_{t_{0}}=\sup _{t \geq t_{0}}\left|x_{t}\right|=\sup _{t \geq t_{0}-\tau}|x(t)| .
$$

Again, together with (3), we will analyze its unperturbed version:

$$
\dot{x}(t)=F\left(x_{t}, 0\right) .
$$

A set $\mathscr{S} \subset \mathscr{C}_{\tau}$ is invariant for the unperturbed system (4) if $X_{t}^{x_{0}, 0} \in \mathscr{S}$ for all $t \in \mathbb{R}_{+}$ and for all $x_{0} \in \mathscr{S}$. Define the distance from a function $\xi \in \mathscr{C}_{\tau}$ to a set $\mathscr{S} \subset \mathscr{C}_{\tau}$ as $\|\xi\|_{\mathscr{S}}=\inf _{\alpha \in \mathscr{S}}|\xi-\alpha|$. 
Let $\mathscr{W} \subset M$ be a set, denote by $\overbrace{\mathscr{W}}$ a subset of $\overline{\mathscr{W}}=\left\{\xi \in \mathscr{C}_{\tau}: \xi(t) \in \mathscr{W} \forall t \in\right.$ $[-\tau, 0]\}$ such that if $\zeta \in \overbrace{\mathscr{W}}$ then $\zeta=X_{\tau}^{\xi, 0}$ for $\xi \in \overline{\mathscr{W}}$. For stability analysis of timedelay systems it is necessary to define a distance to invariant sets in two spaces: in $\mathbb{R}^{n}$ with respect to the set $\mathscr{W}$ and in $\mathscr{C}_{\tau}$ with respect to corresponding invariant set $\overbrace{\mathscr{W}}$ (functions from $\mathscr{C}_{\tau}$ taking values in $\mathscr{W}$ and solutions of (3)). The following stability notions for (3) are considered in this work [10] (for a recent survey on stability tools for time-delay systems see [16]).

Definition 9. The system (3) has the pAG property with respect to the set $\mathscr{W}$ if there exist $\eta \in \mathscr{K}_{\infty}$ and a non-negative real $q$ such that for all $x_{0} \in \mathscr{C}_{\tau}$ and all bounded piecewise continuous inputs $d(\cdot)$ the solutions are defined for all $t \geq 0$ and the following holds:

$$
\limsup _{t \rightarrow+\infty}\left|X\left(t, x_{0} ; d\right)\right|_{\mathscr{W}} \leq \eta\left(\left\|d_{t}\right\|_{0}\right)+q .
$$

If $q=0$, then we say that the AG property holds.

This property can be equivalently stated as

$$
\limsup _{t \rightarrow+\infty}\left\|X_{t}^{x_{0}, d}\right\|_{\overbrace{\mathscr{W}}} \leq \eta\left(\left\|d_{t}\right\|_{0}\right)+q
$$

and it implies that (a subset of) $\overbrace{\mathscr{W}}$ is invariant for (4) if $q=0$.

Definition 10. The system (3) has the pGS property with respect to the set $\mathscr{W}$ if there exist $\beta \in \mathscr{K}_{\infty}$ and $q \geq 0$ such that for all $x_{0} \in \mathscr{C}_{\tau}$ and all bounded piecewise continuous inputs $d(\cdot)$ the following holds for all $t \geq 0$ :

$$
\left|X\left(t, x_{0} ; d\right)\right|_{\mathscr{W}} \leq q+\beta\left(\max \left\{\left\|x_{0}\right\|_{\overbrace{\mathscr{W}}},\left\|d_{t}\right\|_{0}\right\}\right) .
$$

To characterize pAG and pGS properties for a time-delay system (3) the LyapunovRazumikhin approach is used in this work [30,8]. Given a continuous function $x:[-\tau,+\infty) \rightarrow M$ with a $\mathscr{C}^{1}$ function $U: M \rightarrow \mathbb{R}$ denote $U(t)=U(x(t))$, if $x(t)=$ $X\left(t, x_{0} ; d\right)$ is a solution to (3) for some piecewise continuous $d:[-\tau,+\infty) \rightarrow \mathbb{R}^{m}$ and initial condition $x_{0} \in \mathscr{C}_{\tau}$, then the upper right-hand side derivative of $U$ along this solution is

$$
D^{+} U(t)=\limsup _{h \rightarrow 0^{+}} \frac{U(t+h)-U(t)}{h} .
$$

Definition 11. A $\mathscr{C}^{1}$ function $U: M \rightarrow \mathbb{R}$ is a practical ISS-Lyapunov-Razumikhin (ISS-LR) function for (3) if there exist $\mathscr{K}_{\infty}$ functions $\alpha_{1},\left[\alpha_{2}\right], \alpha_{4}, \gamma$ and $\gamma_{U}, \gamma_{U}(s)<s$ for all $s>0$, and scalar $q \geq 0$ [and $c \geq 0$ ] such that

$$
\begin{gathered}
\alpha_{1}\left(|x|_{\mathscr{W}}\right) \leq U(x) \leq\left[\alpha_{2}\left(|x|_{\mathscr{W}}+c\right)\right], \\
U(t) \geq \max \left\{\gamma_{U}\left(\left|U_{t}\right|\right), \gamma\left(\left|d_{t}\right|\right), q\right\} \Rightarrow D^{+} U(t) \leq-\alpha_{4}[U(t)] .
\end{gathered}
$$

If the latter inequality holds for $q=0$, then $U$ is said to be an ISS-LR function. 
Definition 12. The system in (3) is said to be ISS with respect to the set $\mathscr{W}$ if it admits pAG and pGS properties with respect to the set $\mathscr{W}$.

Note that definitions 8 and 12 introduce the same property, but for different classes of systems, (1) and (3), respectively.

Theorem 2. [10] Consider the system (3). Suppose there exists an ISS-LR function $U: M \rightarrow \mathbb{R}$ as in Definition 11. Then the system (3) admits the pAG property from Definition 9 with $\eta(s)=\alpha_{1}^{-1} \circ \gamma(s)$ and the $p G S$ property from Definition 10.

\section{ISS of multistable systems with delayed perturbations}

In this section we consider the robustness of the system (1) with respect to a disturbance $d$, which is dependent on a delayed state. The analysis is conducted under the assumption that the system (1) is ISS with respect to a set $\mathscr{W}$. The proposed approach is illustrated via example of a nonlinear pendulum with delay.

\subsection{Robustness analysis}

If (1) is ISS with respect to the set $\mathscr{W}$, then by Theorem 1 there exists an ISS Lyapunov function $V$ as in Definition 7. From the inequalities $\alpha_{3}\left[0.5 \alpha_{2}^{-1} \circ V(x)\right] \leq$ $\alpha_{3}\left(0.5\left[|x|_{\mathscr{W}}+c\right]\right) \leq \alpha_{3}\left(|x|_{\mathscr{W}}\right)+\alpha_{3}(c)$ we obtain

$$
D V(x) f(x, d) \leq-\alpha_{4}[V(x)]+\gamma(|d|)+\tilde{q},
$$

where $\alpha_{4}(s)=\alpha_{3}\left[0.5 \alpha_{2}^{-1}(s)\right]$ and $\tilde{q}=q+\alpha_{3}(c)$.

Assume that the input $d$ has two terms $d_{1}$ and $d_{2}$, and $d_{2}$ is a function of $x_{t} \in \mathscr{C}_{\tau}$ for some $\tau>0$, i.e.:

$$
d=d_{1}+d_{2}, d_{2}=g\left(x_{t}\right),
$$

where $g$ is a continuous function, $\left|g\left(x_{t}\right)\right| \leq v\left(\left|V_{t}\right|\right)+v_{0}$ for $v \in \mathscr{K}_{\infty}$ and $v_{0} \geq 0$ (here $V_{t}$ denotes a function $V_{t}(\cdot):[-\tau, 0] \rightarrow \mathbb{R}_{+}$defined by $V_{t}(\theta)=V(t+\theta)$ for $\theta \in[-\tau, 0])$. Denote further for simplicity of notation $d=d_{1}$, then (1) is transformed to (3) with

$$
\begin{gathered}
F\left(x_{t}, d_{t}\right)=f\left(x(t), d+g\left(x_{t}\right)\right), \\
D^{+} V(t) \leq-\alpha_{4}(V(t))+\gamma\left(2 v\left(\left|V_{t}\right|\right)+2 v_{0}\right)+\gamma\left(2\left|d_{t}\right|\right)+\tilde{q} .
\end{gathered}
$$

This estimate can be rewritten as follows:

$$
\begin{gathered}
V(t) \geq \max \left\{\hat{\gamma}_{V}\left(\left|V_{t}\right|\right), \hat{\gamma}\left(\left|d_{t}\right|\right), \hat{q}\right\} \Rightarrow D^{+} V(t) \leq-0.5 \alpha_{4}(V(t)), \\
\hat{\gamma}_{V}(s)=\alpha_{4}^{-1}[6 \gamma(4 v(s))], \hat{\gamma}(s)=\alpha_{4}^{-1}[6 \gamma(2 s)], \hat{q}=\alpha_{4}^{-1}\left[6 \tilde{q}+6 \gamma\left(4 v_{0}\right)\right] .
\end{gathered}
$$


It is straightforward to see that if $\hat{\gamma}_{V}(s)<s$ for all $s>0$, then $V$ is an ISS-LR function for (1) with (5), and by Theorem 2 this system possesses pAG and pGS properties.

\subsection{Illustration for a nonlinear pendulum}

Now, the procedure for a robust ISS analysis of a multistable system with delays outlined in Section 4.1 is illustrated via the example of a nonlinear pendulum. First, we prove the assumption made in Section 4.1 that the pendulum is ISS with respect to a set $\mathscr{W}$. Second, a condition for ISS of a pendulum with delay is derived. During our analysis, we also establish almost global attractivity of an equilibrium of a nonlinear pendulum with constant nonzero input. To the best of our knowledge, such result is not available in the literature thus far.

\subsubsection{Delay-free case}

Consider a nonlinear pendulum:

$$
\begin{aligned}
& \dot{x}_{1}=x_{2}, \\
& \dot{x}_{2}=-\Omega^{2} \sin \left(x_{1}\right)-\kappa x_{2}+d,
\end{aligned}
$$

where the state $x=\left[x_{1}, x_{2}\right]$ takes values on the cylinder $M:=\mathbb{S} \times \mathbb{R}, d(t) \in \mathbb{R}$ is an exogenous disturbance, and $\Omega, \kappa$ are constant positive parameters. The total energy of (6) is $H(x)=0.5 x_{2}^{2}+\Omega^{2}\left(1-\cos \left(x_{1}\right)\right)$ and $\dot{H}=x_{2} d-\kappa x_{2}^{2}$. The unperturbed system (6) has two equilibria $[0,0]$ and $[\pi, 0]$ (the former is attractive and the latter one is a saddle-point). Thus, $\mathscr{W}=\{[0,0] \cup[\pi, 0]\}$ is a compact set containing all $\alpha$ - and $\omega$-limit sets of (6) for $d=0$. In addition, it is straightforward to check that $\mathscr{W}$ is decomposable in the sense of Definition 3.

Lemma 1. [10] The system (6) is ISS with respect to the set $\mathscr{W}$.

By using this result it is possible to prove that for a constant input $d$ (with $d<\Omega^{2}$ ) the pendulum still has two steady-state points with similar stability properties.

Lemma 2. [10] Let $d<\min \left\{\Omega^{2}, \sqrt{\frac{\rho \lambda_{\min }(Y)}{2}} \frac{\pi}{\zeta}, 0.5 \sqrt{\varepsilon} \Omega \frac{\pi}{\zeta}, \xi\right\}$ be a constant input in (6), where

$$
\begin{gathered}
\rho=\min \left[\frac{\kappa-\varepsilon}{1+\varepsilon}, \frac{1}{\sqrt{2 \pi}} \frac{\varepsilon \Omega^{2}}{\left(\Omega^{2}+(\kappa+1) \varepsilon\right)}\right], \\
\zeta=\sqrt{\sqrt{2 \pi}\left[\varepsilon \Omega^{-2}+\frac{1}{\kappa-\varepsilon}\right]}, \xi=\frac{2 \sqrt{\Omega^{2}+\kappa \varepsilon}}{\zeta}\left(\frac{\Omega^{2}+(\kappa+1) \varepsilon}{\varepsilon \Omega^{2}}+\frac{1}{\sqrt{2 \pi} \rho}\right)
\end{gathered}
$$

and $0<\varepsilon<\min \{1, \kappa\}$ is a parameter. Then the system has two equilibria, $\left[\arcsin \left(d \Omega^{-2}\right), 0\right]$ and $\left[\pi-\arcsin \left(d \Omega^{-2}\right), 0\right]$. The former one is almost globally attractive. 


\subsubsection{A delayed case study}

Now consider a time-delay modification of (6):

$$
\begin{aligned}
& \dot{x}_{1}(t)=x_{2}(t), \\
& \dot{x}_{2}(t)=-\Omega^{2} \sin \left[x_{1}(t-\tau)\right]-\kappa x_{2}(t)+d(t),
\end{aligned}
$$

where $\tau>0$ is a fixed delay. The unperturbed system (7) with $d(t)=0$ has the same equilibria as (6), i.e. $[0,0]$ and $[\pi, 0]$. The system (7) can be represented as follows:

$$
\begin{aligned}
& \dot{x}_{1}(t)=x_{2}(t) \\
& \dot{x}_{2}(t)=-\Omega^{2} \sin \left[x_{1}(t)\right]-\kappa x_{2}(t)+d(t)+\Omega^{2}\left\{\sin \left[x_{1}(t)\right]-\sin \left[x_{1}(t-\tau)\right]\right\} .
\end{aligned}
$$

By the mean value theorem

$$
\left|\sin \left[x_{1}(t)\right]-\sin \left[x_{1}(t-\tau)\right]\right|=\left|\cos \left[x_{1}(\phi)\right] x_{2}(\phi) \tau\right| \leq\left|x_{2}(\phi)\right| \tau
$$

for some $\phi \in[t-\tau, t]$. Thus, the system (7) can be analyzed as a perturbed nonlinear pendulum with part of the input $d$ dependent on the delay. Using $V$ we obtain for $\mu=\varepsilon \Omega^{-2}+\frac{1}{\kappa-\varepsilon}$ :

$$
D^{+} V(t) \leq-0.5[\kappa-\varepsilon] x_{2}^{2}-0.5 \varepsilon \Omega^{2} \sin ^{2}\left(x_{1}\right)+\mu \Omega^{4} x_{2}^{2}(\phi) \tau^{2}+\mu d^{2} .
$$

It is straightforward to check that

$$
\begin{gathered}
V(x) \leq 0.5[1+\varepsilon] x_{2}^{2}+0.5 \varepsilon \sin ^{2}\left(x_{1}\right)+2\left[\Omega^{2}+\kappa \varepsilon\right], \\
x_{2}^{2} \leq \frac{2}{1-\varepsilon} V(x)+\frac{\varepsilon}{1-\varepsilon}
\end{gathered}
$$

for $0<\varepsilon<\min \{1, \kappa\}$, then for $\rho=\min \left\{\frac{\kappa-\varepsilon}{1+\varepsilon}, \Omega^{2}\right\}$

$$
\begin{aligned}
D^{+} V(t) & \leq-\rho\left\{V(t)-2\left[\Omega^{2}+\kappa \varepsilon\right]\right\}+\mu \Omega^{4} x_{2}^{2}(\phi) \tau^{2}+\mu d^{2} \\
& \leq-\rho\left\{V(t)-2\left[\Omega^{2}+\kappa \varepsilon\right]\right\}+\frac{\mu \Omega^{4}}{1-\varepsilon} \tau^{2}[2 V(\phi)+\varepsilon]+\mu d^{2} .
\end{aligned}
$$

Therefore,

$V(t) \geq \frac{6}{\rho} \max \left\{2 \frac{\mu \Omega^{4}}{1-\varepsilon} \tau^{2}\left|V_{t}\right|, 2 \rho\left[\Omega^{2}+\kappa \varepsilon\right]+\frac{\mu \Omega^{4}}{1-\varepsilon} \tau^{2} \varepsilon, \mu d^{2}\right\} \Rightarrow D^{+} V(t) \leq-0.5 \rho V(t)$

and $V$ is an ISS-LR function for (7) provided that

$$
\frac{12}{\rho} \frac{\mu \Omega^{4}}{1-\varepsilon} \tau^{2}<1
$$


The inequality (8) is a delay-dependent stability condition for (7), which is always satisfied for a sufficiently small delay $\tau$. The set of asymptotic attraction for (7) can be evaluated from (4.2.2).

Remark 1. If we assume that $\max \left\{0, \frac{\kappa-\Omega^{2}}{1+\Omega^{2}}\right\}<\varepsilon<\min \{1, \kappa\}$, then $\min \left\{\frac{\kappa-\varepsilon}{1+\varepsilon}, \Omega^{2}\right\}=$ $\frac{\kappa-\varepsilon}{1+\varepsilon}$ and the condition (8) can be rewritten as follows:

$$
\tau^{2}<\frac{12}{\Omega^{2}} \frac{1-\varepsilon}{1+\varepsilon} \frac{(\kappa-\varepsilon)^{2}}{\varepsilon(\kappa-\varepsilon)+\Omega^{2}} .
$$

Since the functions $\frac{1-\varepsilon}{1+\varepsilon}$ and $\frac{(\kappa-\varepsilon)^{2}}{\varepsilon(\kappa-\varepsilon)+\Omega^{2}}$ are decreasing for $\varepsilon \in\left(\max \left\{0, \frac{\kappa-\Omega^{2}}{1+\Omega^{2}}\right\}, \min \{1, \kappa\}\right)$, selecting $\varepsilon=\max \left\{0, \frac{\kappa-\Omega^{2}}{1+\Omega^{2}}\right\}+\varepsilon$ for a sufficiently small $\varepsilon>0$ (if $\kappa>\Omega^{2}$ then the optimal choice is $\varepsilon=\frac{\kappa-\Omega^{2}}{1+\Omega^{2}}$ ) optimizes the value of the admissible delay $\tau$ to

$$
\tau^{*}=\frac{\kappa-\varepsilon}{\Omega} \sqrt{\frac{1-\varepsilon}{1+\varepsilon} \frac{12}{\varepsilon(\kappa-\varepsilon)+\Omega^{2}}},
$$

i.e. for any $\tau<\tau^{*}$ the system (7) admits $V$ as an ISS-LR function.

\section{Application to a microgrid composed of two droop-controlled inverters with delay}

In this section the theoretical results of Section 3 are applied to our main motivating application: a droop-controlled microgrid with delays. In particular, we are interested in conditions for ISS of such systems. In order to tackle this problem, we proceed along the lines detailed in Section 4. The analysis is conducted under a reasonable assumption of constant voltage amplitudes. Then, a lossless droopcontrolled microgrid formed by two inverters with delay can be modeled as [35]:

$$
\begin{aligned}
\dot{\theta}(t) & =\omega_{1}(t)-\omega_{2}(t), \\
\tau_{P_{1}} \dot{\omega}_{1}(t) & =-\omega_{1}(t)-k_{P_{1}} a_{12} \sin \left[\theta\left(t-\tau_{d_{1}}\right)\right]+c_{1}+\delta_{1}(t), \\
\tau_{P_{2}} \dot{\omega}_{2}(t) & =-\omega_{2}(t)+k_{P_{2}} a_{12} \sin \left[\theta\left(t-\tau_{d_{2}}\right)\right]+c_{2}+\delta_{2}(t),
\end{aligned}
$$

where $\theta(t) \in[0,2 \pi)$ is the phase difference between the inverters, $\omega_{1}(t), \omega_{2}(t) \in \mathbb{R}$ are the time-varying frequencies of the inverters; $\tau_{d_{1}}>0$ and $\tau_{d_{2}}>0$ are delays caused by the digital controls required to implement the droop controls; $\tau_{P_{1}}>0$, $\tau_{P_{2}}>0, k_{P_{1}}>0, k_{P_{2}}>0, a_{12}>0, c_{1}$ and $c_{2}=-\frac{k P_{2}}{k P_{1}} c_{1}$ are constant parameters; the disturbances $\delta_{1}(t)$ and $\delta_{2}(t)$ represent additional model uncertainties. We say that a solution of (9) is phase-locked if $\theta(t)=\theta_{0}$ is constant $\forall t \in \mathbb{R}_{+}$for some $\theta_{0} \in[0,2 \pi)$ [15]. If this property holds asymptotically, i.e., for $t \rightarrow+\infty$, we speak about an asymptotic phase-locking. 
As sumption. $\tau_{d_{1}}=\tau_{d_{2}}=\tau>0$.

Under this assumption, define the new coordinates:

$$
x_{1}=\theta, x_{2}=\omega_{1}-\omega_{2}, x_{3}=\frac{k_{P_{2}} \tau_{P_{1}}}{k_{P_{1}} \tau_{P_{2}}} \omega_{1}+\omega_{2} .
$$

Then the system (9) can be rewritten as follows:

$$
\begin{aligned}
& \dot{x}_{1}(t)=x_{2}(t) \\
& \dot{x}_{2}(t)=-b_{1} x_{2}(t)+b_{2} x_{3}(t)-a^{2} \sin \left[x_{1}(t-\tau)\right]+d_{1}(t), \\
& \dot{x}_{3}(t)=-b_{3} x_{3}(t)+b_{4} x_{2}(t)+d_{2}(t)
\end{aligned}
$$

where

$$
\begin{gathered}
b_{1}=\tau_{P_{1}}^{-1}+\left(\tau_{P_{2}}^{-1}-\tau_{P_{1}}^{-1}\right)\left(1+\frac{k_{P_{2}} \tau_{P_{1}}}{k_{P_{1}} \tau_{P_{2}}}\right)^{-1} \frac{k_{P_{2}} \tau_{P_{1}}}{k_{P_{1}} \tau_{P_{2}}}, b_{2}=\left(\tau_{P_{2}}^{-1}-\tau_{P_{1}}^{-1}\right)\left(1+\frac{k_{P_{2}} \tau_{P_{1}}}{k_{P_{1}} \tau_{P_{2}}}\right)^{-1}, \\
b_{3}=\tau_{P_{1}}^{-1}+\left(\tau_{P_{2}}^{-1}-\tau_{P_{1}}^{-1}\right)\left(1+\frac{k_{P_{2}} \tau_{P_{1}}}{k_{P_{1}} \tau_{P_{2}}}\right)^{-1}, b_{4}=\left(\tau_{P_{2}}^{-1}-\tau_{P_{1}}^{-1}\right)\left(1+\frac{k_{P_{2}} \tau_{P_{1}}}{k_{P_{1}} \tau_{P_{2}}}\right)^{-1} \frac{k_{P_{2}} \tau_{P_{1}}}{k_{P_{1}} \tau_{P_{2}}} \\
a=\sqrt{\left(k_{P_{1}} \tau_{P_{1}}^{-1}+k_{P_{2}} \tau_{P_{2}}^{-1}\right) a_{12}}, \\
d_{1}(t)=\left(\tau_{P_{1}}^{-1}+\tau_{P_{2}}^{-1} \frac{k_{P_{2}}}{k_{P_{1}}}\right) c_{1}+\tau_{P_{1}}^{-1} \delta_{1}(t)-\tau_{P_{2}}^{-1} \delta_{2}(t), d_{2}(t)=\frac{k_{P_{2}}}{k_{P_{1}} \tau_{P_{2}}} \delta_{1}(t)+\tau_{P_{2}}^{-1} \delta_{2}(t) .
\end{gathered}
$$

In [10] it has been also assumed that $\tau_{P_{1}}=\tau_{P_{2}}=\tau_{P}>0$. The stability analysis above is based on the assumption that $b_{1}>0$ and $b_{3}>0$, which we imposed without loss of generality. If $\tau_{P_{2}}>\tau_{P_{1}}$ and the coefficients $b_{1}<0$ or $b_{3}<0$ for the given values of $k_{P_{1}}$ and $k_{P_{2}}$, then the above equations can be rewritten to have the term $\tau_{P_{1}}^{-1}-\tau_{P_{2}}^{-1}$ instead of $\tau_{P_{2}}^{-1}-\tau_{P_{1}}^{-1}$ by flipping the indices. Thus, the system (9) is decomposed into two interrelated subsystems: (10), (11) and (12). The variable $x_{3}$ converges asymptotically to zero if $b_{4} x_{2}+d_{2}=0$ (then asymptotically the frequencies $\omega_{1}$ and $\omega_{2}$ are locked), moreover this subsystem is ISS with respect to the input $b_{4} x_{2}+d_{2}$, with the ISS asymptotic gain $\left|b_{4} x_{2}+d_{2}\right| \rightarrow\left|x_{3}\right|$ being equal to $b_{3}^{-1}$ (this simple result can be obtained using an ISS Lyapunov function $\left.V_{3}\left(x_{3}\right)=0.5 x_{3}^{2}\right)$.

The dynamics (10), (11) have the form of (7) for $d=d_{1}+b_{2} x_{3}$ and, as it has been established above, have pAG and pGS properties from definitions 9 and 10 respectively if condition (8) is satisfied, which for (10), (11) takes the form:

$$
\tau^{2}<\frac{12}{a^{2}} \frac{1-\varepsilon}{1+\varepsilon} \frac{\left(b_{1}-\varepsilon\right)^{2}}{\varepsilon\left(b_{1}-\varepsilon\right)+a^{2}}
$$

for $0<\varepsilon<\min \left\{1, b_{1}\right\}$, and the asymptotic gain $d^{2} \rightarrow V$ is $\varepsilon a^{-2}+\frac{1}{b_{1}-\varepsilon}$. Therefore, under the small-gain condition 


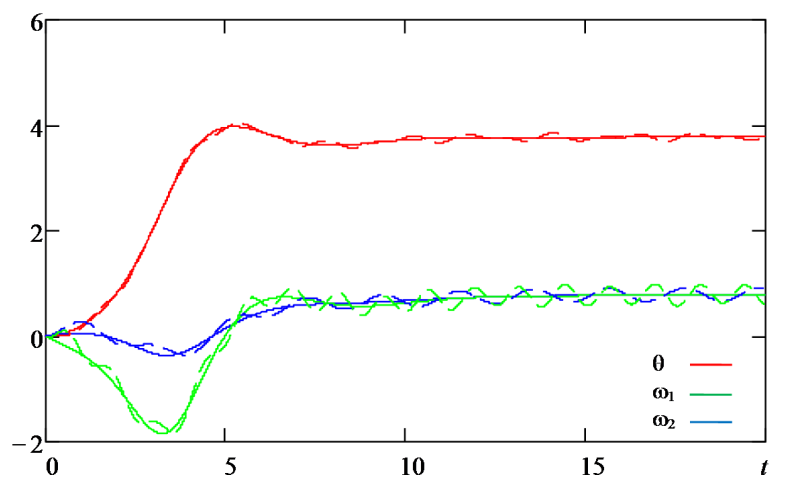

Fig. 1 Simulation results for the system (9). The solid lines show the state trajectories for the case $d_{1}(t)=d_{2}(t)=0$. The dashed lines correspond to the case $d_{1}(t)=0.8 \sin (3 t), d_{2}(t)=0.9 \sin (5 t)$.

$$
b_{3}^{2} b_{4}^{2} b_{2}^{2}\left(\varepsilon a^{-2}+\frac{1}{b_{1}-\varepsilon}\right)<1
$$

and for a sufficiently small delay $\tau$ verifying (13) the system (10), (11), (12) is ISS with respect to inputs $d_{1}$ and $d_{2}$. In that case the inverters will demonstrate a phaselocking behavior. According to [29], a good estimate of the overall delay introduced by the digital control is $\tau=1.75 T_{S}{ }^{1}$, where $T_{S}=1 / f_{S}$ and $f_{S} \in \mathbb{R}_{>0}$ is the switching frequency of the inverter. Since usually $f_{S} \in[5,20] \mathrm{kHz}[17], \tau$ is reasonably small in most practical applications. Hence, the condition (13) may be satisfied for most practical choices of parameters in (9).

The analysis is illustrated in a simulation example with the following set of parameters for the system (9): $\tau_{P_{1}}=2, \tau_{P_{2}}=1, k_{P_{1}}=10, k_{P_{2}}=20, a_{12}=0.1, c_{1}=0.2$ and $\tau=0.05$. Conditions (13) and (14) are satisfied for $\varepsilon=0.5 \min \left\{1, b_{1}\right\}$. The simulation results are shown in Fig. 1. The solid lines represent the state $\left(\theta, \omega_{1}, \omega_{2}\right)^{T}$ trajectories for the case $d_{1}(t)=d_{2}(t)=0$, and the dashed lines correspond to $d_{1}(t)=0.8 \sin (3 t), d_{2}(t)=0.9 \sin (5 t)$. The phase-locking phenomenon is observed in these simulation results.

\section{Conclusions}

Sufficient conditions for ISS of multistable systems with delay have been presented. The conditions are formulated using Lyapunov-Razumikhin functions. The potential of the approach has been illustrated by demonstrating several robustness properties for a nonlinear pendulum with delay. Furthermore, it has been shown that asymptotic phase-locking in a lossless droop-controlled microgrid formed by two inverters with delays can be analyzed based on a perturbed pendulum model. By exploiting

${ }^{1}$ The overall delay reduces to $\tau=1.5 T_{S}$ if no moving average function for the measurement is used [29]. 
this fact, a delay-dependent condition for ISS of such a microgrid has been presented.

Acknowledgements This work was supported in part by the Government of Russian Federation (Grant 074-U01) and the Ministry of Education and Science of Russian Federation (Project 14.Z50.31.0031).

\section{References}

1. D. Angeli. An almost global notion of input-to-state stability. IEEE Trans. Automatic Control, 49:866-874, 2004.

2. D. Angeli and D. Efimov. On input-to-state stability with respect to decomposable invariant sets. In Proc. 52nd IEEE Conference on Decision and Control, Florence, 2013.

3. D. Angeli and D. Efimov. Characterizations of input-to-state stability for systems with multiple invariant sets. Automatic Control, IEEE Transactions on, PP(99):1-13, 2015.

4. D. Angeli, J.E. Ferrell, and E.D. Sontag. Detection of multistability, bifurcations and hysteresis in a large class of biological positive-feedback systems. Proc. Natl. Acad. Sci. USA, 101:1822-1827, 2004.

5. D. Angeli and L. Praly. Stability robustness in the presence of exponentially unstable isolated equilibria. IEEE Trans. Automatic Control, 56:1582-1592, 2011.

6. M.C. Chandorkar, D.M. Divan, and R. Adapa. Control of parallel connected inverters in stand-alone AC supply systems. IEEE Transactions on Industry Applications, 29(1):136 143, jan/feb 1993.

7. M. Chaves, T. Eissing, and F. Allgower. Bistable biological systems: A characterization through local compact input-to-state stability. IEEE Trans. Automatic Control, 45:87-100, 2008.

8. S. Dashkovskiy and L. Naujok. Lyapunov-Razumikhin and Lyapunov-Krasovskii theorems for interconnected ISS time-delay systems. In Proc. 19th International Symposium on Mathematical Theory of Networks and Systems(MTNS), pages 1179-1184, Budapest, 2010.

9. S.N. Dashkovskiy, D.V. Efimov, and E.D. Sontag. Input to state stability and allied system properties. Automation and Remote Control, 72(8):1579-1614, 2011.

10. D. Efimov, J. Schiffer, and R. Ortega. Robustness of delayed multistable systems with application to droop-controlled inverter-based microgrids. International Journal of Control, 89(5):909-918, 2016.

11. Denis Efimov, Romeo Ortega, and Johannes Schiffer. ISS of multistable systems with delays: application to droop-controlled inverter-based microgrids. In Proc. ACC 2015, Chicago, 2015.

12. D.V. Efimov and A.L. Fradkov. Oscillatority of nonlinear systems with static feedback. SIAM Journal on Optimization and Control, 48(2):618-640, 2009.

13. Xi Fang, Satyajayant Misra, Guoliang Xue, and Dejun Yang. Smart grid - the new and improved power grid: a survey. Communications Surveys \& Tutorials, IEEE, 14(4):944-980, 2012.

14. H. Farhangi. The path of the smart grid. IEEE Power and Energy Magazine, 8(1):18 -28, january-february 2010.

15. Alessio Franci, Antoine Chaillet, Elena Panteley, and Françoise Lamnabhi-Lagarrigue. Desynchronization and inhibition of Kuramoto oscillators by scalar mean-field feedback. Mathematics of Control, Signals, and Systems, 24(1-2):169-217, 2012.

16. Emilia Fridman. Tutorial on Lyapunov-based methods for time-delay systems. European Journal of Control, 20:271-283, 2014.

17. T.C. Green and M. Prodanovic. Control of inverter-based micro-grids. Electric Power Systems Research, Vol. 77(9):1204-1213, july 2007. 
18. J. Guckenheimer and P. Holmes. Structurally stable heteroclinic cycles. Math. Proc. Camb. Phil. Soc., 103:189-192, 1988.

19. J Guerrero, P Loh, Mukul Chandorkar, and T Lee. Advanced control architectures for intelligent microgrids - part I: Decentralized and hierarchical control. IEEE Transactions on Industrial Electronics, 60(4):1254-1262, 2013.

20. N. Hatziargyriou, H. Asano, R. Iravani, and C. Marnay. Microgrids. IEEE Power and Energy Magazine, 5(4):78 -94, july-aug. 2007.

21. Christopher M. Kellett, Fabian R. Wirth, and Peter M. Dower. Input-to-state stability, integral input-to-state stability, and unbounded level sets. In Proc. 9th IFAC Symposium on Nonlinear Control Systems, pages 38-43, Toulouse, 2013.

22. Osman Kukrer. Discrete-time current control of voltage-fed three-phase PWM inverters. IEEE Transactions on Power Electronics, 11(2):260-269, 1996.

23. R.H. Lasseter. Microgrids. In IEEE Power Engineering Society Winter Meeting, 2002, volume 1, pages $305-308$ vol.1, 2002.

24. J.A.P. Lopes, C.L. Moreira, and A.G. Madureira. Defining control strategies for microgrids islanded operation. IEEE Transactions on Power Systems, 21(2):916 - 924, may 2006.

25. Dragan Maksimovic and Regan Zane. Small-signal discrete-time modeling of digitally controlled PWM converters. IEEE Transactions on Power Electronics, 22(6):2552-2556, 2007.

26. P. Monzn and R. Potrie. Local and global aspects of almost global stability. In Proc. 45th IEEE Conf. on Decision and Control, pages 5120-5125, San Diego, USA, 2006.

27. Ulrich Münz and Diego Romeres. Region of attraction of power systems. In Estimation and Control of Networked Systems, volume 4, pages 49-54, 2013.

28. Z. Nitecki and M. Shub. Filtrations, decompositions, and explosions. American Journal of Mathematics, 97(4):1029-1047, 1975.

29. Thomas Nussbaumer, Marcelo Lobo Heldwein, Guanghai Gong, Simon D Round, and Johann W Kolar. Comparison of prediction techniques to compensate time delays caused by digital control of a three-phase buck-type PWM rectifier system. IEEE Transactions on Industrial Electronics, 55(2):791-799, 2008.

30. P. Pepe, I. Karafyllis, and Z.-P. Jiang. On the Liapunov-Krasovskii methodology for the ISS of systems described by coupled delay differential and difference equations. Automatica, 44(9):2266-2273, 2008.

31. R. Rajaram, U. Vaidya, and M. Fardad. Connection between almost everywhere stability of an ODE and advection PDE. In Proc. 46th IEEE Conf. Decision and Control, pages 5880-5885, New Orleans, 2007.

32. A. Rantzer. A dual to Lyapunov's stability theorem. Syst. Control Lett., 42:161-168, 2001.

33. Joan Rocabert, Alvaro Luna, Frede Blaabjerg, and Pedro Rodriguez. Control of power converters in AC microgrids. IEEE Transactions on Power Electronics, 27(11):4734-4749, Nov 2012.

34. V.V. Rumyantsev and A.S. Oziraner. Stability and stabilization of motion with respect to part of variables. Nauka, Moscow, 1987. [in Russian].

35. Johannes Schiffer, Romeo Ortega, Alessandro Astolfi, Jörg Raisch, and Tevfik Sezi. Conditions for stability of droop-controlled inverter-based microgrids. Automatica, 2014. Accepted.

36. Johannes Schiffer, Romeo Ortega, Christian A Hans, and Jörg Raisch. Droop-controlled inverter-based microgrids are robust to clock drifts. In American Control Conference (ACC), pages 2341-2346. IEEE, 2015.

37. J. W. Simpson-Porco, F. Dörfler, and F. Bullo. Synchronization and power sharing for droopcontrolled inverters in islanded microgrids. Automatica, 49(9):2603 - 2611, 2013.

38. S. Smale. Differentiable dynamical systems. Bull. Amer. Math. Soc., 73:747-817, 1967.

39. E. D. Sontag and Y. Wang. On characterizations of input-to-state stability with respect to compact sets. In Proc IFAC Non-Linear Control Systems Design Symposium, (NOLCOS '95), pages 226-231, Tahoe City, CA, 1995.

40. E.D. Sontag. On the input-to-state stability property. European J. Control, 1:24-36, 1995.

41. E.D. Sontag and Y. Wang. New characterizations of input-to-state stability. IEEE Trans. Autom. Control, 41(9):1283-1294, 1996. 
42. G.-B. Stan and R. Sepulchre. Analysis of interconnected oscillators by dissipativity theory. IEEE Trans. Automatic Control, 52:256-270, 2007.

43. Andrew R. Teel. Connections between Razumikhin-type theorems and the ISS nonlinear small gain theorem. IEEE Trans. Automat. Control, 43(7):960-964, 1998.

44. R. van Handel. Almost global stochastic stability. SIAM J. Control and Optimization, 45(4):1297-1313, 2006.

45. Pravin P Varaiya, Felix F Wu, and Janusz W Bialek. Smart operation of smart grid: Risklimiting dispatch. Proceedings of the IEEE, 99(1):40-57, 2011. 